\title{
Laparoscopic repair of a perforated duodenal ulcer: another use of a round ligament flap
}

\author{
Kevin Allart ${ }^{1,2}$, Flavien Prevot ${ }^{1,2}$, Lionel Rebibo ${ }^{1,2}$, Jean-Marc Regimbeau ${ }^{1,2}$ \\ ${ }^{1}$ Department of Digestive Surgery, Amiens University Hospital, Amiens, France \\ ${ }^{2}$ Simplification des Soins des Patients Complexes (SSPC) - Clinical Research Unit, University of Picardie Jules Verne, Amiens, France \\ Videosurgery Miniinv 2018; 13 (4): 542-545 \\ DOI: https://doi.org/10.5114/wiitm.2018.76070
}

\begin{abstract}
Management of a perforated duodenal ulcer is most commonly performed by laparoscopy and consists of suture of the perforation after performing lavage of the peritoneal cavity. In most cases, a flap is created, and an omental flap is usually the preferred choice because of its simplicity and its proximity to the site of duodenal perforation. However, in some cases, the greater omentum cannot be used due to the severity of peritonitis or due to previous surgical removal. We report a laparoscopic technique for surgical repair of a perforated duodenal ulcer using a round ligament flap. The present manuscript and the associated video highlight some important technical aspects to easily perform this procedure.
\end{abstract}

Key words: laparoscopy, duodenal ulcer, perforated ulcer, falciform ligament flap.

\section{Introduction}

At the present time, management of a perforated duodenal ulcer is most commonly performed by laparoscopy [1] after selection of patients based on the Boey score [2]. The surgical technique consists of lavage of the peritoneal cavity after taking bacteriological and mycological samples, followed by suture of the perforation with an on-lay omental patch, and finally drainage of the operative site [3]. Closure of perforated ulcers with omental patches should be performed systematically, as it decreases mortality and morbidity rates [4]. The simplest technique for laparoscopic repair usually requires the use of an omental flap [5]. However, in some cases, the greater omentum cannot be used as a patch to close the perforation.

\section{Operative technique}

We report our surgical technique for laparoscopic repair of a perforated duodenal ulcer located on the anterior wall of the first part of the duodenum (Photos $1 \mathrm{~A}, \mathrm{~B}$ ). The patient was positioned in the French position; the surgeon stood between the patient's legs with the first assistant to the right. A $12 \mathrm{~mm}$ trocar was initially inserted just above the umbilicus by the open Hasson technique. A $5 \mathrm{~mm}$ trocar was then inserted through the right rectus abdominis, a $10 \mathrm{~mm}$ trocar was inserted through the left rectus abdominis and a $5 \mathrm{~mm}$ trocar was inserted at the epigastrium under laparoscopic vision. Initial exploration of the peritoneal cavity revealed generalized chemical peritonitis (Photos 2 A, B). Bacteriological and mycological samples were taken. Peritoneal lavage was performed and all fibrin membranes were removed. Exploration of the abdominal cavity was then performed in order to visualize the perforation (Photo $2 \mathrm{C}$, Figure $1 \mathrm{~A}$ ). The duodenal ulcer was sutured by two absorbable surgical sutures (Polysorb 3-0, Covidien France SAS, Elancourt, France) (Figure $1 \mathrm{~B}$ ).

\section{Address for correspondence}

Prof. Jean-Marc Regimbeau, Department of Digestive Surgery, Amiens University Hospital, Avenue René Laennec, 80000 Amiens, France, phone: +33322668301, e-mail: regimbeau.jean-marc@chu-amiens.fr 

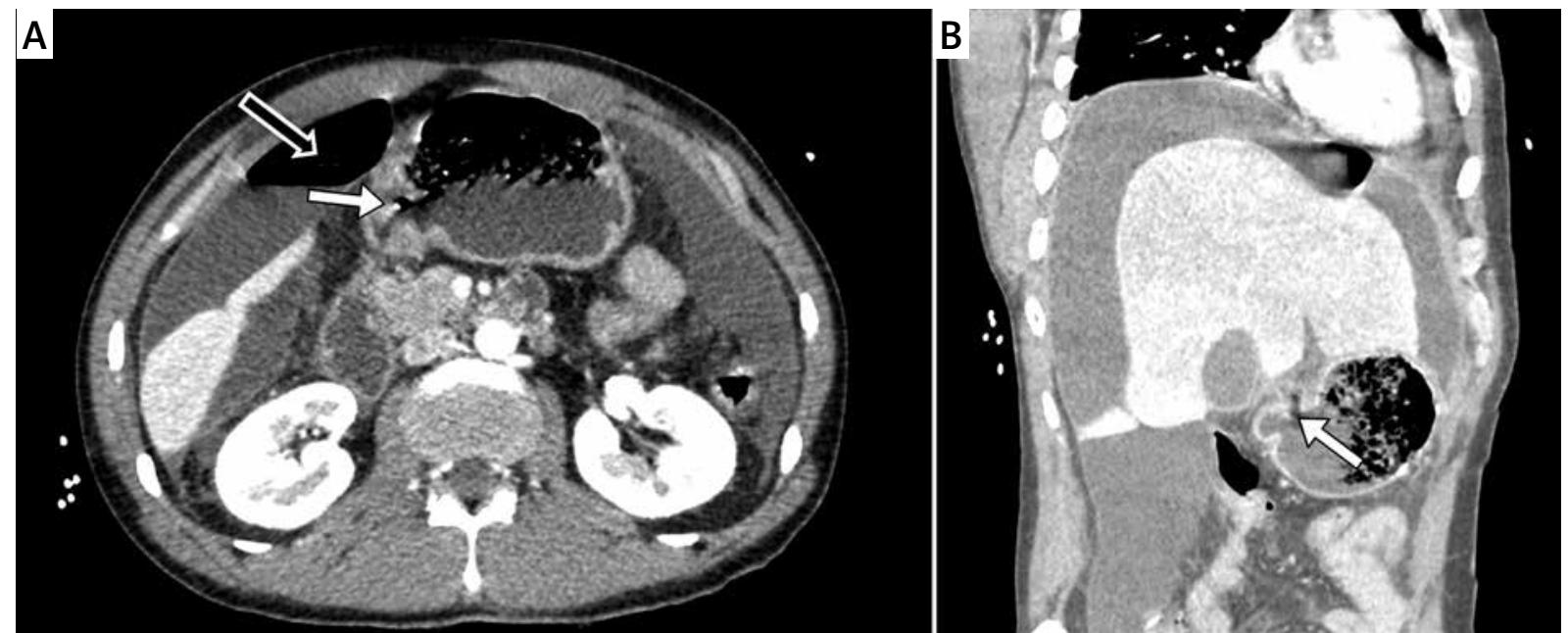

Photo 1. Preoperative abdominal CT scan of perforated duodenal ulcer. A - Axial abdominal CT scan showing perforation of the wall of the first part of the duodenum (white arrow) and the presence of pneumoperitoneum (black arrow), B - coronal abdominal CT scan showing perforation located on the anterior surface of the first part of the duodenum (white arrow)

The ends of the suture were left long inside the abdominal cavity with the needles carefully positioned on the greater omentum to avoid organ injury. A methylene blue test was then performed to confirm the absence of leak. As an omental flap could not be performed due to severe peritonitis, a round ligament flap was preferred. The falciform ligament was released to allow tension-free mobili-
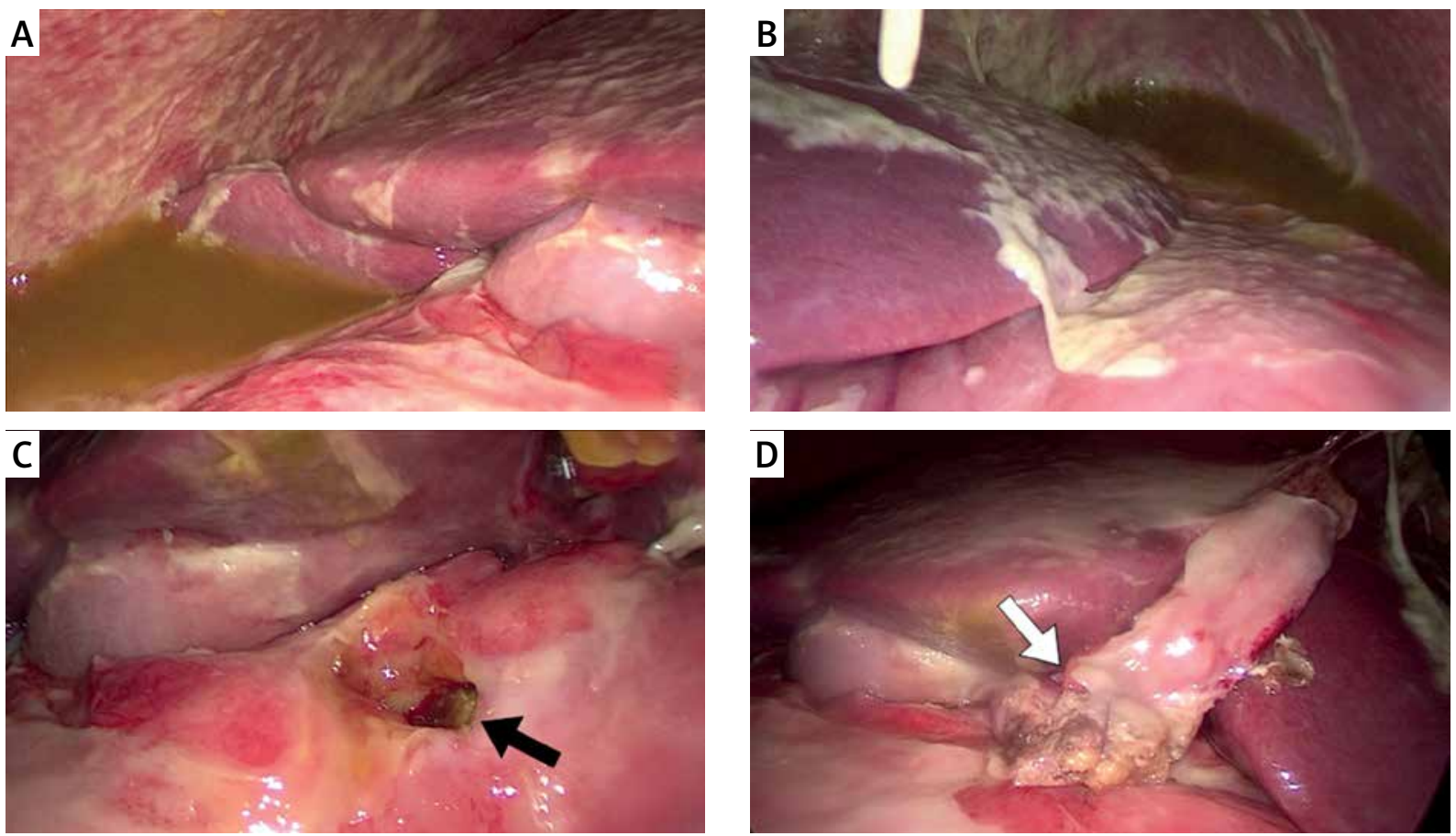

Photo 2. Intraoperative findings. A - Chemical peritonitis in the right upper quadrant, $\mathbf{B}$ - chemical peritonitis in the left upper quadrant, $\mathbf{C}$ - intraoperative view showing perforation of the first part of the duodenum (black arrow), D - intraoperative view showing falciform ligament patch over the perforated duodenal ulcer (white arrow) 


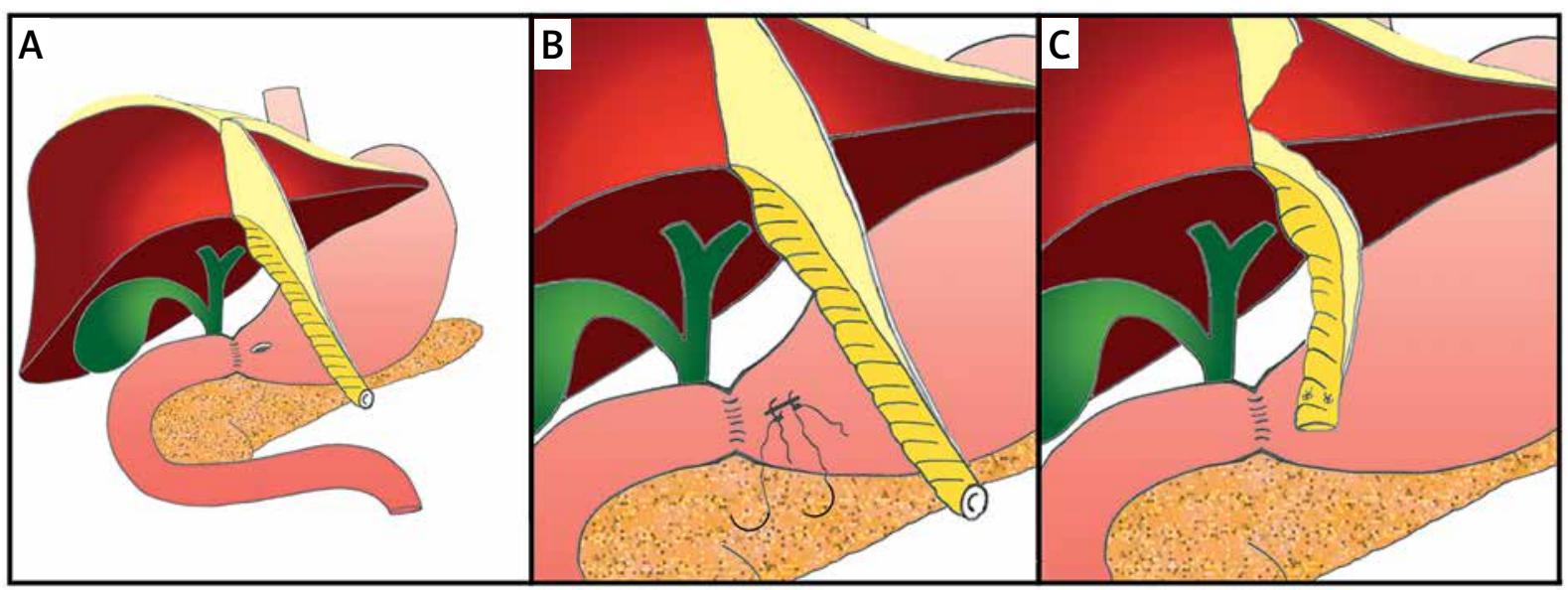

Figure 1. Diagrams of the various steps of perforated ulcer repair using a falciform ligament patch. A - Prepyloric perforation and mobilization of the falciform ligament, $\mathbf{B}$ - direct suture of the perforation leaving long stay sutures, $\mathbf{C}$ - suture of the falciform ligament flap

zation, first on the peritoneal side and then on the hepatic side. The stay sutures were then recovered and used to fix the round patch over the duodenal ulcer (Photo 2 D, Figure $1 \mathrm{C}$ ). At the end of the surgical procedure, a suction drain was placed through the $5 \mathrm{~mm}$ right trocar and positioned close to the round ligament flap (Video).

Postoperative management consisted of removal of the nasogastric tube on postoperative day one and proton pump inhibitor and antibiotic therapy. An oral methylene blue test to ensure the absence of a leak was performed on postoperative day 3, allowing oral refeeding. The drainage was then removed on postoperative day 4 .

\section{Discussion}

The use of the falciform ligament has been regularly described to manage difficult intra-abdominal situations, such as venous reconstruction [6], prevention of pancreatic fistula or protection of vessels to decrease the risk of pseudoaneurysm in the presence of a pancreatic fistula [7], or hemostasis of a ruptured liver tumor [8]. Fry et al. described the first use of a falciform ligament in a perforated peptic ulcer in 1978 [9]. In 1996, Munro et al. published a series of 6 patients treated for a perforated duodenal ulcer by laparoscopic suture with a falciform ligament flap [10].

Duodenal ulcer repair is usually associated with an omental flap to protect the suture of the duodenal perforation. However, in some cases, this technique is not possible, but the combination of a flap with duodenal ulcer repair seems to be associated with a lower incidence of postoperative complications [4]. Using a round or falciform ligament to replace the traditional omental patch is interesting, in such cases, to complete the direct closure of the ulcer.

We have used the round ligament flap in some cases of duodenal ulcer with good outcomes, and this patch is technically feasible and quite simple. In some cases, no direct closure of the perforation was performed, but the round ligament was simply sutured over the duodenal ulcer (Video). We have also routinely used this patch to protect vessels as part of laparoscopic pancreatoduodenectomy.

\section{Conclusions}

A round ligament patch is technically feasible by laparoscopy and seems to be a valuable alternative to an omental patch for patients with a history of omental resection or in the presence of major peritoneal inflammation preventing the use of an omental patch.

\section{Video link}

https://www.dropbox.com/sh/tjbav439x8qkljk/ AADWcriOHGZPRQPowCFiMPpDa?dl=0

\section{Conflict of interest}

The authors declare no conflict of interest. 


\section{References}

1. Tan S, Wu G, Zhuang Q, et al. Laparoscopic versus open repair for perforated peptic ulcer: a meta analysis of randomized controlled trials. Int J Surg 2016; 33: 124-32.

2. Lee FYJ, Leung KL, Lai BSP, et al. Predicting mortality and morbidity of patients operated on for perforated peptic ulcers. Arch Surg 2001; 136: 90-4.

3. Mouly C, Chati R, Scotté M, Regimbeau JM. Therapeutic management of perforated gastro-duodenal ulcer: literature review. J Visc Surg 2013; 150: 333-40.

4. Turner WW, Thompson WM, Thal ER. Perforated gastric ulcers: a plea for management by simple closures. Arch Surg 1988; 123: 960-4.

5. Rebibo L, Darmon I, Regimbeau JM. Laparoscopic surgical technique for perforated duodenal ulcer. J Visc Surg 2016; 153: 127-33.

6. Ikegami T, Shimada M, Imura S, et al. Beneficial use of the round ligament as a patch graft for vena cava reconstruction. $J$ Hepatobiliary Pancreat Surg 2008; 15: 581-4.

7. Ray S, Sanyal S, Ghatak S, et al. Falciform ligament flap for the protection of the gastroduodenal artery stump after pancreaticoduodenectomy: a single center experience. J Visc Surg 2016; 153: 9-13.

8. Fujino Y, Ku Y, Suzuki Y, Kuroda Y. Successful haemostasis of a ruptured liver tumour using flaps of gallbladder serosa and the falciform ligament. Eur J Surg 2001; 167: 552-3.

9. Fry DE, Richardson JD, Flint LM. Closure of an acute perforated peptic ulcer with the falciform ligament. Arch Surg 1978; 113: 1209-10.

10. Munro WS, Bajwa F, Menzies D. Laparoscopic repair of perforated duodenal ulcers with a falciform ligament patch. Ann R Coll Surg Engl 1996; 78: 390-1.

Received: 16.08 .2016 , accepted: 28.04 .2018 . 